\title{
Ärztliche Schweigepflicht
}

\author{
K. Burchardt ${ }^{1}$, P. Burchardt ${ }^{2}$, H. Baumgärtel ${ }^{3}$ \\ 1 Richter am Bundessozialgericht \\ 2 Chefarzt Urologische Klinik, DRK-Krankenhaus Seepark, Langen-Bremerhaven \\ ${ }^{3}$ Chefarzt Urologische Klinik, Krankenhaus Siloah, Hannover
}

\section{Zusammenfassung}

Die Schweigepflicht ist die höchste Standes- und Rechtspflicht des Arztes. Sie schützt die Persönlichkeit des Patienten und umfaßt medizinische Bereiche, aber auch private Informationen. Gemäß $\$ 203$ Strafgesetzbuch macht sich derjenige strafbar, der die ärztliche Schweigepflicht durchbricht, ausführlich geregelt ist sie in der „Berufsordnung für die deutschen Ärzte“.

\section{Summary}

The professional discretion is the physician's highest class and legal duty. It protects the patient's personality and includes medical ranges, but also private information. According to $\$ 203$ Penal Code you are liable to punishment in case you will break the medical professional discretion. It is regulated in detail in the „Berufsordnung für die deutschen Ärzte“.

\section{Key words}

Professional discretion - Right of refusal to give evidence - Obligation to report

\section{Ärztliche Schweigepflicht}

Die seit Hippokrates als Kernstück ärztlicher Berufsethik bezeichnete ärztliche Schweigepflicht (1) ermöglicht und garantiert das Vertrauensverhältnis zwischen Patienten und Arzt. Sie ist die höchste ärztliche Standes- und Rechtspflicht und dient dem Schutz der Persönlichkeitssphäre des Patienten. Die Einhaltung des Arztgeheimnisses ist unverzichtbar für die Ausübung des ärztlichen Berufs; nur in Kenntnis der Verschwiegenheit wird der Patient seine gesundheitlichen Beschwerden offen darlegen. Deshalb beschränkt sich die Schweigepflicht nicht bloß auf medizinische Tatsachen; sie ist vielmehr umfassend und erstreckt sich z. B. auch auf sonstige Informationen, etwa Familien- und Partnerprobleme (2).

Den hohen Stellenwert der Schweigepflicht hat der Gesetzgeber dadurch zum Ausdruck gebracht, da $ß$ sich strafbar macht, wer das Arztgeheimnis unbefugt durchbricht (\$203 Strafgesetzbuch). Wesentliche Grundsätze zur ärztlichen Schweigepflicht enthält die „Berufsordnung für die deutschen Ärzte" (3). Schließlich ist die Einhaltung der ärztlichen Schweigepflicht durch den Behandlungsvertrag zwischen Arzt und Patienten abgesichert, zu dessen Inhalt auch die Verpflichtung zur Verschwiegenheit gehört. Die Wahrung des ärztlichen Berufsgeheimnisses enthält eine fast unübersehbare Fülle von

Akt. Urol. 24 (1993) 160-162

(C) Georg Thieme Verlag Stuttgart · New York
Problemen, die im Nachfolgenden nur in den wichtigsten Grundzügen angedeutet werden können. Die Schweigepflicht gilt grundsätzlich gegenüber jedermann, also auch unter Ärzten und selbstverständlich gleichfalls den Familienangehörigen des Arztes gegenüber. Die Schweigepflicht dauert im bisherigen Umfang über den Tod des Patienten hinaus.

Entscheidend für die Befugnis, Patientengeheimnisse an ärztliche Kollegen zu offenbaren, ist deren Einbeziehung in das Behandlungsgeschehen. Das bedeutet, daß der Krankenhausarzt gegenüber dem Assistenzpersonal seiner Abteilung und dem vom Patienten konsiliarisch oder zur Weiterbehandlung in Anspruch genommenen anderen Arzt nicht zur Verschwiegenheit verpflichtet ist. Einzuhalten ist das Arztgeheimnis allerdings gegenüber allen anderen, in die Mitbehandlung nicht eingebundenen Kollegen. Aufgehoben ist die Schweigepflicht auch, wenn der Patient nach stationärem Aufenthalt an den einweisenden Arzt zurücküberwiesen wird.

Gegenüber Familienangehörigen des Patienten gilt grundsätzlich ebenso die Schweigepflicht. Für die Annahme einer schlüssig erklärten Einwilligung des Patienten zur Offenbarung an Familienangehörige empfiehlt sich eine gewisse Zurückhaltung.

Mit Einschränkungen gilt auch bei Minderjährigen die Pflicht zur Verschwiegenheit gegenüber den Erziehungsberechtigten. Hier kollidiert die Schweigepflicht mit dem im Elternrecht wurzelnden und durch den 
Erziehungszweck gerechtfertigten Bedürfnis auf Information. Bei Minderjährigen mit ausreichender Reife kann es im Interesse des Kindes durchaus geboten sein, den Eltern gegenüber zu schweigen (4).

Die Durchbrechung der ärztlichen Schweigepflicht ist in der Regel gerechtfertigt durch das Einverständnis des Patienten, durch die Abwägung zum Schutze eines höherwertigen Rechtsgutes oder durch bestimmte gesetzliche Offenbarungs- und Meldepflichten.

Neben einer ausdrücklich erklärten Entbindung von der Schweigepflicht kann die Zustimmung zur Offenbarung durch schlüssiges Verhalten erfolgen. Eine solche Zustimmung durch schlüssiges Verhalten dient auch als Legitimation für das Arztgespräch mit dem Patienten am Krankenbett, wenn Dritte (Mitpatienten - aber nicht Besucher!) der Unterredung folgen können. Diese mit Zurückhaltung anzunehmende - Zustimmung erstreckt sich jedoch nicht auf gänzlich überraschende Inhalte.

Die Schweigepflicht besteht auch gegenüber der Krankenhausverwaltung, deren Angestellte im Gegensatz zu den ärztlichen Mitarbeitern nicht ärztliches Hilfspersonal sind. Daher hat die Zuleitung ärztlichen Schriftwechsels an die Krankenhausverwaltung zu unterbleiben. Es ist ebenfalls rechtlich bedenklich, ärztliche Berichte zentral in einem Schreibbüro schreiben zu lassen. Ein entsprechendes Einverständnis des Patienten kann man nicht unterstellen. Die Weitergabe von Abrechnungsunterlagen an die privatärztliche Verrechnungsstelle bedarf der vorherigen ausdrücklichen schriftlichen Einwilligung des Patienten. Die Abtretung einer ärztlichen Honorarforderung an eine solche Verrechnungsstelle, die zum Zwecke der Rechnungsstellung und Einziehung unter Übergabe der Abrechnungsunterlagen erfolgt, ist wegen Verletzung des bürgerlichen Gesetzbuches (\$134) nichtig, wenn der Patient ihr nicht vorher schriftlich zustimmt (5). Dies gilt auch, wenn der Arzt die Abrechnung der abgetretenen Forderung selbst vorgenommen hat (6).

Bei Veräußerung einer Arztpraxis mit Übergabe der Patienten- und Beratungskartei muß der Arzt die Einwilligung der betroffenen Patienten in eindeutiger und unmißverständlicher Weise einholen (7).

Bei der Aufbewahrung von Krankenunterlagen im Krankenhaus muß ebenfalls die ärztliche Schweigepflicht gewahrt bleiben. Werden die Unterlagen in einem Zentralarchiv abgelegt, ist durch geeignete Maßnahmen sicherzustellen, daß diese Unbefugten nicht zugänglich sind.

Weitere gesetzliche Offenbarungspflichten enthalten die Vorschriften des Sozialgesetzbuches - Gesetzliche Krankenversicherung - (\$\$ 294 ff SGB V). Darin ist die Verpflichtung und Befugnis des Kassenarztes vorgesehen, bestimmte Angaben, die für die Erfüllung der Aufgaben der Krankenkassen sowie der Kassenärztlichen Vereinigungen notwendig sind, aufzunehmen und diesen Stellen nach näherer Maßgabe dieser Vorschriften mitzuteilen. Dem Verlangen privater Versicherungsunternehmen, Patientendaten zu übermitteln, darf nur bei zwei- felsfreier Einwilligung des Patienten entsprochen werden. Probleme hinsichtlich des Geheimnisschutzes erwachsen aus den immer weniger überschaubaren Forschungsaktivitäten und dem zunehmenden Einsatz der elektronischen Datenverarbeitung. Sowohl die Speicherung als auch die Darstellung von Krankheitsfällen in wissenschaftlichen Abhandlungen sind zu anonymisieren oder bedürfen der ausdrücklichen Zustimmung der Betroffenen (8).

Bei übergesetzlichem Notstand (Gefahr für Leib, Leben oder Freiheit von Patienten oder anderen Personen) kann die Weitergabe von Patientendaten an Dritte gerechtfertigt sein. Auch das Verhalten eines mit AIDS infizierten Patienten gegenüber seiner Umwelt kann eine Information des Lebensgefährten rechtfertigen. Hier sollte der Arzt sogar von einer Verpflichtung zur Warnung des Partners ausgehen, wenn dieser bei demselben Arzt wie der Infizierte in Behandlung ist (9). Zu betonen bleibt jedoch, da $\beta$ es sich - bis auf die zuletzt geschilderte Ausnahme - um ein Recht und nicht um eine Pflicht des Arztes zur Offenbarung handelt.

Im Gegensatz dazu verpflichten bestimmte Anzeige- und Meldepflichten den Arzt zum Geheimnisbruch. Der Arzt, der von einem geplanten Mord oder Totschlag erfährt, muß dies melden. Auch andere Kapitalverbrechen (Raub, räuberische Erpressung, Freiheitsberaubung) bedürfen der Meldung ( 138 Strafgesetzbuch).

Weitere Meldepflichten ergeben sich bei bestimmten Krankheiten nach dem Bundesseuchengesetz (\$3) und dem Geschlechtskrankheitengesetz (\$\$12,13). Grundsätzlich ist jeder Arzt auch verpflichtet, Angaben über Geburten und Todesfälle zu machen. Gegenüber Unfallversicherungsträgern ist der behandelnde Arzt zur Auskunft über die Befunde und den Zustand eines durch Arbeitsunfall Verletzten verpflichtet ( $\$ 1543$ der Reichsversicherungsordnung).

Im gerichtlichen Verfahren wird der ärztlichen Schweigepflicht dadurch Rechnung getragen, da $B$ dem Arzt ein Zeugnisverweigerungsrecht zusteht über alles, was ihm in seiner Eigenschaft als Arzt anvertraut oder sonst bekanntgeworden ist ( $\$ 383$ Zivilprozeßordnung, $\$ \$ 53,53$ a Strafprozeßordnung (10). Das Zeugnisverweigerungsrecht ist damit das prozessuale Korrelat der Schweigepflicht.

Im Strafprozeß unterliegen Untersuchungsbefunde und andere Krankenunterlagen dem Beschlagnahmeverbot (\$97 Strafprozeßordnung). Das Verbot hat den Zweck, daß das Zeugnisverweigerungsrecht nicht durch Beschlagnahme umgangen wird. Deshalb dürfen im Strafverfahren gegen einen Patienten Krankenunterlagen beim behandelnden Arzt nicht beschlagnahmt werden.

Die unbefugte Verletzung der ärztlichen Schweigepflicht ist unter Strafe gestellt; sie wird allerdings nur auf Antrag des Betroffenen verfolgt. Die Verletzung der Schweigepflicht kann zivilrechtliche Schadensersatzansprüche wegen Verletzung des Behandlungsvertrages nach sich ziehen. 


\section{Literatur}

1 Laufs: Arztrecht, 4. Aufl., Verlag C. H. Beck, München (1988) 135

2 Narr: Ärztliches Berufsrecht, 2. Aufl., Stand: September 1989 , Deutscher Ärzte-Verlag, Köln, Band 2, RdNr. 747

3 Deutsches Ärzteblatt, Ausgabe B (1988) B-2547

4 Narr: Arztliches Berufsrecht, 2. Aufl., Stand: September 1989, Deutscher Ärzte-Verlag, Köln, Band 2, RdNr. 754

5 Bundesgerichtshof (BGH) in Neue Juristische Wochenschrift (NJW), S. 2955; Oberlandesgericht (OLG) Bremen in NJW 1992, S. 757

6 OLG Oldenburg in NJW (1992) 758

7 BGH in NJW (1992) 737

8 s. $\$ 2$ Absatz 7 der Berufsordnung

9 Tegtmeyer-Metzdorf/Grundmann: Krankenhausarzt und Recht, Bibliomed. Mediz. Verlagsgesellschaft mbH, Melsungen (1990) 112

$10 \mathrm{BGH}$ in NJW (1985) 2203, 2204
Prof: Dr. med. P. Burchardt

DRK-Krankenhaus Seepark

Urologische Klinik

2857 Langen-Debstedt 